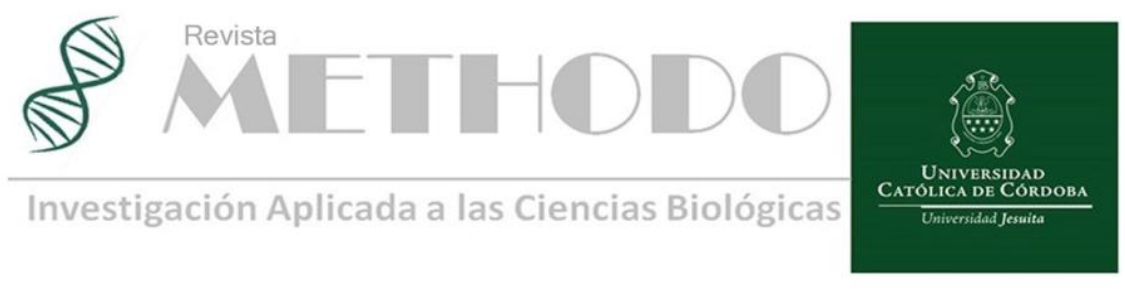

ARTICULO ORIGINAL Methodo 2019;4(3):71-80 https://doi.org/10.22529/me.2019.4(3)02

\title{
Consejo genético y detección de vías moleculares en pacientes con cáncer hereditario
}

\section{Genetic advice and molecular pathways detection in patients with hereditary cancer}

\author{
Daniel Lerda ${ }^{1}$, Patricia Pellicioni ${ }^{1}$, Marta Biaggi ${ }^{1}$, Jorge Labrador ${ }^{2}$, Edith Illescas $^{2}$, Santiago Bella ${ }^{3}$, José \\ Llugdar $^{3}$, Matías Cortes ${ }^{3}$. \\ 1 Universidad Católica de Córdoba Facultad de Ciencias de la Salud, Clínica Universitaria Reina Fabiola. Laboratorio de Genética Molecular. \\ 2 Laboratorio de Biología Molecular, Universidad Maimónides, Buenos Aires, Argentina \\ 3 Universidad Católica de Córdoba Facultad de Ciencias de la Salud, Clínica Universitaria Reina Fabiola. Servicio de Oncología. \\ Correspondencia: Daniel Enrique Lerda. Laboratorio de Genética Molecular - Clínica Universitaria Reina Fabiola. Oncativo 1248 -X5004FHP- Córdoba, \\ Argentina; email: daniellerda@curf.ucc.edu.ar.
}

\section{Resumen}

INTRODUCCIÓN: El cáncer de mama es la primera causa de muerte de la mujer en la Argentina, con una incidencia estimada de más de 19.000 casos nuevos por año. Dentro de estos, el tipo de cáncer hereditario más común es el de mama/ovario hereditario, provocado por mutaciones en los genes BRCA 1 (Breast cáncer) y BRCA 2. A su vez el cáncer colorrectal es la segunda causa de muerte en Argentina, con una incidencia estimada de más de 11.000 casos nuevos por año.

OBJETIVO: de la presente investigación es evaluar la utilidad de la realización de los estudios genéticos en personas con cáncer hereditario en el contexto del consejo genético, con un asesoramiento antes y después de realizarse la prueba genética

POBLACIÓN Y MÉTODOS: Se estudiaron 34 mujeres con diagnóstico de cáncer de mama/ovario y 31 pacientes de ambos sexos con diagnóstico de cáncer colorrectal (CCR). En las mujeres se analizaron los genes $B R C A 1$ y 2 por secuenciación de próxima generación (NSG) y grandes rearreglos de los genes BRCA 1 y 2 por amplificación de sonda dependiente de la ligadura multiplex (MLPA). En las personas de ambos sexos se determinó la Inestabilidad de Microsatelites(IMS), el análisis de mismatch repair (MMR) por MLPA y la mutación del gen $B R A F$ (Protooncogen $B$-Raf)

RESULTADOS: Los resultados mostraron que las pacientes con cáncer de mama / ovario con antecedentes familiares tienen un alto porcentaje de $B R C A$ negativo. En cuanto a los cambios fenotípicos, el más predominante en este estudio, fue el subtipo triple negativo y la paciente con BRCA 2 positivo presentó este fenotipo. Con respecto al estudio del cáncer de colon detectamos cuatro pacientes con IMS-alta y mutación del V600E del gen BRAF. Cuando se les realizó el análisis de MLPA en los genes MSH6, MLH1, MSH2 y PMS2 a los efectos de establecer la diferencia entre CCR y síndrome de Lynch, los resultados fueron negativos, por lo tanto, estos pacientes fueron diagnosticados como CCR esporádico.

CONCLUSIONES: Como lo demuestra este trabajo, para el consejo genético, el estudio de vías moleculares en pacientes con cáncer hereditario es un instrumento de ayuda para la valoración del riesgo génico.

Palabras claves: $B R C A 1$ y 2 , Inestabilidad de Microsatelites, $B R A F$ cáncer hereditario de mama /ovario y colon, consejo genético.

Revista Methodo: Investigación Aplicada a las Ciencias Biológicas. Universidad Católica de Córdoba. Jacinto Ríos 571 Bo Gral. Paz. X5004FXS. Córdoba. Argentina. Tel.: (54) 3514517299 / Correo: methodo@ucc.edu.ar / Web: methodo.ucc.edu.ar | ARTICULO ORIGINAL Methodo 2019;4(3):71-80 


\section{Abstract}

INTRODUCTION: Breast cancer is the leading cause of death of women in Argentina, with a estimated incidence of more than 19,000 new cases per year. Among these, the most common type of inherited cancer is the breast /ovarian caused by mutations in the BRCAl and BRCA 2 genes. At the same time, the cancer colorrectal is the second cause of death in Argentina, with an estimated incidence of more than 11,000 new cases per year.

OBJECTIVE: of the present investigation is to evaluate the usefulness of the realization of the genetic studies in people with hereditary cancer in the context of genetic counseling, with a advice before and after the genetic test.

POPULATIONS AND METHODS: We studied 34 women diagnosed with breast / ovarian cancer and 31 patients of both sexes with diagnosis of colorectal cancer (CRC). In women, the BRCA 1 and 2 genes by next generation sequencing (NSG) and large rearrangements of $B R C A 1$ and 2 genes by probe amplification dependent on multiplex ligation(MLPA). The instability of microsatellites (IMS) was determined in people of both sexes, the analysis of mismatchrepair (MMR) by MLPA and the mutation of the BRAF gene.

RESULTS:

There results showed that patients with breast / ovarian cancer with a history family members have a high percentage of negative $B R C A$. As for the changes phenotypic, the most predominant in this study, was the triple negative subtype and the patient with positive $B R C A 2$ presented this phenotype. With respect to the study of colon cancer detected four patients with IMS-high and mutation of the V600E of the BRAF gene. When the MLPA analysis was performed on the MSH6, MLH1, MSH2 genes and PMS2, in order to established difference between CRC and Lynch syndrome, the results were negative and therefore these patients were diagnosed as sporadic CRC.

CONCLUSIONS: As this work demonstrates, for him genetic counseling, the study of pathways molecules in patients with hereditary cancer is a helpful instrument for risk assessment.

Keywords: molecular pathways, $B R C A 1$ y 2 , microsatellite instability, $B R A F$, hereditary cancer of breast/ovary and colon, genetic advice.

\section{Introducción}

El cáncer es la enfermedad más común y aparece con mayor frecuencia a edades avanzadas, como consecuencia de alteraciones genéticas producidas a lo largo de la vida bajo la influencia de factores tales como los ambientales. Sin embargo, los recientes avances en genética molecular han conllevado a la identificación de genes de alta penetrancia responsables de un número importante de enfermedades.

Actualmente, se dispone de los test-DNA predictivos para tres grupos de enfermedades: enfermedades neurogenéticas, cánceres hereditarios y enfermedades cardiovasculares. Entre ellos, los estudios genéticos se dirigen, principalmente, a tres síndromes de cáncer hereditarios: síndrome de cáncer de mama y ovario, síndrome de cáncer colorrectal y poliposis adenomatosa familiar. El cáncer de mama es la primera causa de muerte de la mujer en la Argentina, con una incidencia estimada de más de 19.000 casos nuevos por año, lo cual representa el $16,8 \%$ del total de incidencia de cáncer en Argentina. El más común de los síndromes de cáncer de mama hereditario es el síndrome de cáncer de mama/ovario hereditario, provocado por mutaciones en los genes BRCAl (OMIM 113705) y BRCA 2 (OMIM 600185). La presencia de historia familiar de cáncer de mama es un factor de riesgo importante y demostrado, pero hay que considerar que puede ser secundaria a factores ambientales y/o genéticos compartidos entre miembros de una misma familia. Estudios de cohorte han demostrado que tener un familiar de primer grado, sea madre o hermana, con cáncer de mama incrementa en 1,5-2 el riesgo de una mujer de desarrollar cáncer de mama ${ }^{1}$. Este riesgo es superior si la edad de diagnóstico es inferior a los 50 años o si es un cáncer de mama bilateral. El cáncer colorrectal (CCR) es la segunda causa de muerte en Argentina, con una incidencia estimada de más de 11.000 casos nuevos por año. El $25 \%$ de los casos se encuentran agrupados en familias, en las cuales la predisposición a desarrollar la enfermedad se encuentra aumentada. Entre 3 y $8 \%$ de los casos son producidos por mutaciones heredables en genes puntuales y constituyen los distintos síndromes de cáncer colorrectal hereditario. El más común de los síndromes de cáncer de colon hereditario es el síndrome de Lynch o síndrome de cáncer colorrectal hereditario no poliposo. El síndrome de Lynch, también conocido como cáncer colorrectal hereditario no asociado a poliposis (CCHNP), es una enfermedad hereditaria con herencia autosómica dominante y 
es a la predisposición genética a sufrir cáncer colorrectal más frecuente, junto con otras manifestaciones extracolónicas como neoplasias de endometrio, ovario, estómago, intestino delgado, tracto hepatobiliar, tracto urinario superior, cerebro y piel.

El mejor conocimiento de las bases genéticas del cáncer, junto con la identificación de diversos genes asociados a síndromes de predisposición hereditaria al cáncer y el desarrollo de diversas técnicas de laboratorio, ha abierto la posibilidad de ofrecer consejo genético especializado a individuos y familias en situación de riesgo. La identificación de estos individuos y familias permite, además de una valoración individualizada del riesgo de desarrollar cáncer, recomendar estrategias de prevención y cribado adecuadas al riesgo estimado cuya efectividad en la reducción de la incidencia y mortalidad por cáncer haya sido demostrada.

El consejo genético de predisposición hereditaria al cáncer es el proceso de información y comunicación no directiva a las personas $\mathrm{o}$ familias en situación de riesgo de cáncer, en lo que se refiere a la probabilidad de presentar o transmitir a su descendencia una determinada susceptibilidad genética a desarrollar una neoplasia, sobre sus implicaciones, sobre la posibilidad de realizar un diagnóstico molecular y sobre cuáles son las medidas disponibles para la prevención y el diagnóstico precoz.

El proceso de valoración de riesgo puede incluir o no la realización de un estudio genético. El estudio genético es un instrumento de ayuda para la valoración del riesgo una vez que, por criterios clínicos, éste se considera alto. Son éstos los casos en que se recomienda el consejo y asesoramiento genéticos. Los estudios genéticos pueden permitir mejorar el manejo del riesgo de cáncer, y reducir la incertidumbre y la ansiedad de desarrollar un cáncer tanto en la persona afectada como en su familia.

Los estudios genéticos deben efectuarse siempre en el contexto del consejo genético, con un asesoramiento antes y después de realizarse la prueba genética, discutiendo sus limitaciones y los posibles riesgos y beneficios, no sólo de la prueba en cuestión, sino también de las opciones para la detección precoz y de las medidas disponibles para la reducción del riesgo.

El objetivo de la presente investigación es el análisis de la utilidad de los estudios genéticos en el contexto del consejo genético, con un asesoramiento antes y después de realizarse la prueba genética a los efectos de actualizar el estado del conocimiento científico respecto a los cánceres hereditarios de presentación más común, recomendando pautas de actuación en los ámbitos de diagnóstico, prevención y tratamiento sobre la base del nivel de evidencia científica existente.

\section{Material y métodos}

\section{Sujetos del estudio}

Se estudiaron 34 pacientes mujeres con diagnóstico de cáncer de mama/ovario cuyas edades fueron entre 22 y 74 años y 31 pacientes de ambos sexos con edades entre 26 y 77 años con diagnóstico de cáncer colorrectal (CCR), que concurrieron al servicio de Oncología de la Clínica Universitaria Reina Fabiola (CURF). Los pacientes fueron derivados a consejo genético entre junio de 2016 y agosto de 2018 por presentar criterios clínicos de sospecha de predisposición genética a ambos tipos de cáncer. A los individuos de alto riesgo de síndrome hereditario se les indicó la realización del estudio genético. Previamente se les explicó sus beneficios, limitaciones, riesgos, y se confirmó que lo hubieran entendido correctamente y que estuvieran en condiciones de hacerse el estudio. Los pacientes que aceptaron firmaron un consentimiento informado el cual fue previamente aprobado por el comité de Ética de la Clínica Universitaria Reina Fabiola. Se utilizaron muestras de sangre periférica, como tejido normal y muestras de tumor embebido en parafina.

\section{Unidad de Consejo Genético}

A todos los pacientes estudiados se le realizó un asesoramiento antes y después de realizarse la prueba genética, discutiendo sus limitaciones y los posibles riesgos y beneficios, no sólo de la prueba en cuestión, sino también de las opciones para la detección precoz y de las medidas disponibles para la reducción del riesgo.

\section{Extracción del ADN}

La extracción de ADN a partir de sangre periférica para los estudios de BRCA 1 y 2

e IMS se realizó empleando el kit Wizard Genomic DNA Purification Kit de Promega. siguiendo el protocolo indicado por el fabricante. Se aisló el ADN genómico y después de lo cual se midió con el espectrofotómetro Nanodrop-1000 (Termofisher) estableciendo la concentración de ADN en 1-2 ul de muestras, con un buen grado de pureza. De la muestra de taco de parafina se realizó un corte que fue coloreado con Hematoxilina-Eosina para confirmar por microscopia óptica la presencia de la zona tumoral, y uno a dos cortes en blanco se usaron para la extracción del ADN genómico. La extracción y 
purificación del ADN genómico se llevó a cabo con kit comercial de extracción (ReliaPrep FFPE gDNAMiniprepSystem -Promega), siguiendo el protocolo indicado por el fabricante.

\section{Secuenciación de los Genes BRCA1 y BRCA2 por NextGenerationSequencing (NSG)}

Se emplearon $10 \mathrm{ng}$ de $\mathrm{ADN}$ genómico por reacción para la generación de librerías mediante el kit AmpliSeq Library Kit 2.0. Se amplificaron las regiones codificantes y las zonas intrónicas adyacentes de los genes BRCA1 y BRCA2, en tres ${ }^{2}$ conjuntos de 167 amplicones. La secuenciación se llevó a cabo con el secuenciador masivo PGM (Life Technologies), obteniéndose una cobertura media promedio en las regiones analizadas del 90,87 al $97.57 \mathrm{X}$, estando el $100 \%$ de las bases cubiertas por encima de 20X. Las variantes que se generaron, tras un alineamiento contra el genoma de referencia (UCSC hg19) e identificadas por Torrent Suite 5.0 (Life Technologies), se anotaron de acuerdo a las secuencias de referencia BRCA1: NM_007294.3 y BRCA2: NM_000059.39, según las recomendaciones del Human Genome Variation Society (HGVS) ${ }^{3}$ utilizando la adenina del codón de iniciación de traducción ATG como nucleótido +1 . La evaluación de variantes se realizó consultando las bases de datos específicas para los genes BRCA 1 y 2; HGMD, UMD, BIC, LOVD, Clin Var, ARUP y HCI Data Base. La clasificación final de las variantes noveles se realiza de acuerdo con las directrices de $\mathrm{ACMG}^{2}$, clasificándolas como; patogénicas, probablemente patogénicas, significancia Incierta (VUS), probablemente benignas y benignas. Todas las variantes patogénicas, probablemente patogénicas o VUS, no identificadas previamente se confirmaron mediante amplificación por PCR convencional y secuenciación de Sanger.

\section{Análisis de grandes rearreglos de los genes BRCA 1 y BRCA 2 por Multiplex Ligation-Dependent Probe Amplification(MLPA).}

La técnica de MLPA se realizó utilizando BRCAl KIT P002-C2 probemix y P045 KIT-B3 $B R C A 2 /$ probemixCHEK2 (MRC-Holland, Amsterdam, Netherlands) para los genes BRCA1 y BRCA2. El DNA de los pacientes (75ng, $5 \mu \mathrm{L})$ y tres controles fueron desnaturalizados durante 5 minutos a $98^{\circ} \mathrm{C}$. Se añadió $3 \mu \mathrm{L}$ del mix de hibridación, se calentó a $95^{\circ} \mathrm{C}$ durante 1 minuto y se incubó a $60^{\circ} \mathrm{C}$ durante toda la noche (16 horas). La ligación se realizó con la enzima termoestable Ligasa-65 (32 $\mu$ Lmezcla de Ligasa-
$65)$ durante 20 minutos a $54^{\circ} \mathrm{C}$. Tras la inactivación de la ligasa ( 5 minutos a $\left.98^{\circ} \mathrm{C}\right)$ los productos de ligación se amplificaron por PCR, mediante un oligonucleótido marcado con 6FAM. La PCR consistió en 35 ciclos (30 segundos a $60^{\circ} \mathrm{C}$ y 60 segundos a $72^{\circ} \mathrm{C}$ ). La mezcla de $1 \mu \mathrm{L}$ de los fragmentos resultantes, $0,2 \mu \mathrm{L}$ de standard LIZ-500 y $9 \mu \mathrm{L}$ de formamida altamente desionizada se incubó durante 3 minutos a $90^{\circ} \mathrm{C}$. Tras enfriar a $4^{\circ} \mathrm{C}$ en hielo, la muestra se analizó en un secuenciador capilar ABI 3500 mediante el software Genescan (Applied Biosystems, Foster City, CA). Para el análisis de los resultados se importaron los datos al software Coffalyser v14, teniendo en cuenta el modelo de secuenciador, tamaño de marcador, tipo de sonda y lote de MLPA.

\section{Análisis de la Inestabilidad de Microsatélites}

El análisis de la IMS en los tumores colorrectales se realizó utilizando tejido tumoral y sangre periférica (tejido normal) con los siguientes marcadores: BAT 25 4q (c KIT), BAT 26 2p 16.3 - 21 (h MSH2), D5S346 5q 21-22 (APC), D17S250 17q 11.2-12 (Mfd 15 CA) BRCA 1, D2S123 2p 16 (h MSH2). Cada marcador microsatelital fue amplificado por PCR con primers específicos fluorocromados correspondientes a panel de microsatélites de los genes reparadores del mismatch del ADN (MMR) MSH2 y MLH1. Se realizó el análisis por fragmentos de los productos amplificados con analizador genético ABI Prism 310, comparando la sangre periférica (tejido normal) con el tejido tumoral verificando el perfil de cada microsatélite. Los tumores que presentaron más de (dos o más) microsatélites inestables (con variación en el número de alelos y/o alteración en el patrón de migración) fueron considerados como tumores con IMS alta, aquellos que tuvieron solo uno fueron considerados como IMS-baja y los tumores que presentaron todos los microsatélites normales (wild type) se consideran IMS estables ${ }^{4}$.

\section{Análisis MMR por MLPA}

Se utilizaron los kits comerciales: P072-C1 MSH6, P003-A2 MLH1/MSH2 y P008-C1 PMS2 de MRC-Holland. Los fragmentos obtenidos se analizaron por secuenciador automático. Se estudiaron los rearreglos genómicos, duplicaciones y deleciones, para los genes MSH6, MLH1, MSH2 y PMS2. El análisis se realizó empleando el software Coffalyser. 


\section{Análisis de BRAF}

Se realizó la amplificación del ADN genómico de la región correspondiente al exón 15 del gen BRAF (NCBI Ref. Seq. NG_007873.1), mediante reacción en cadena de la polimerasa (PCR), con el par de cebadores (primers).Para comprobar la integridad de la muestra se realizó la detección en simultaneo del gen constitutivo K-RAS. La amplificación por PCR se hizo en un volumen final de $25 \mu$, utilizando $2,5 \mu \mathrm{l}$ de ADN, $200 \mu \mathrm{M}$ de cada dNTPs, 0,5 U de GoTaq DNA Polymerasa (Promega), $5 \mu$ l buffer GoTaq $5 \mathrm{x}$ conteniendo 1,5 $\mathrm{mM}$ de $\mathrm{MgCl} 2$ (Promega), $0,5 \mu \mathrm{lde} \mathrm{MgCl} 2$ y 0,16 $\mu \mathrm{M}$ de los cebadores forward y reverse. Las condiciones de amplificación fueron: un ciclo inicial de desnaturalización a $94{ }^{\circ} \mathrm{C}$ durante 5 minutos, seguido de 35 ciclos de; desnaturalización a $94{ }^{\circ} \mathrm{C}$ durante 45 segundos, alineamiento (annealing) a $58{ }^{\circ} \mathrm{C}$ durante 45 segundos y extensión de 45 segundos a $72{ }^{\circ} \mathrm{C}$ con una extensión final de 5 minutos a $72{ }^{\circ} \mathrm{C}$. Se verificó el producto de PCR esperado mediante electroforesis en gel de agarosa al 1,8\% y visualización en transiluminador UV, aislando la banda de interés de $224 \mathrm{pb}$. Para la purificación del fragmento de ADN, se empleó el sistema Extraction Kit Wizard Sv Gel and PCR Clean Up System (Promega). Se realizó la reacción de secuencia del eluido con Big Dye Terminator v1.1 (Applied Biosystems), en ambas direcciones por separado y con los mismos cebadores empleados en la reacción de PCR. El producto se purificó en columnas de Sephadex (Centri Sep Spin Columns -Princenton Separations-), y la secuenciación se llevó a cabo en un analizador ABI Prism310 Genetic Analyser (Applied Biosystems). Por último, se analizó el electroferograma del fragmento secuenciado comparándolo contra la secuencia de referencia para detectar la mutación puntual en el codón V600.

\section{Resultados}

Los individuos que concurrieron a consejo genético derivados del servicio de Oncología de la Clínica Universitaria Reina Fabiola poseían una alta probabilidad de ser portadores de una mutación, es decir, eran individuos que tenían una agregación familiar de cáncer significativa o un diagnóstico de cáncer a una edad más precoz de la habitual.

Los resultados de los estudios de pacientes con cáncer de mama/ovario que concurrieron al consejo genético y que en virtud de sus antecedentes se les solicitó test genético, muestran que el tipo de tumor predominante fue el triple negativo 22/34 (64,7 \%) (se caracteriza por la ausencia de expresión de los receptores de

estrógeno, de progesterona y del receptor 2 del factor de crecimiento epidérmico humano HER2), en cambio el Luminal A presentó 6/34 (17,64 \%), el Luminal B 1/34 (2,94\%) y cinco pacientes presentaron cáncer de ovario 5/34 (14,70\%). El paciente $7,1 / 34(2,94 \%)$ presentó la mutación del BRCA 2 (Tabla 1). En este, se ha detectado en el exón 11 del gen BRCA2 la deleción en heterocigosis, del nucleótido $\mathrm{T}$ en la posición genómica chr13:32914438, variante c.5946delTp.Ser1982Argfs*22 generando el corrimiento del marco de lectura (frameshift), la cual se confirmó mediante la secuenciación Sanger convencional. Este corrimiento produce en el posición nucleotídica 2003 un codón de parada prematuro (codón stop) dando lugar a un transcripto (mRNA) truncado, por el alelo con la variante frameshift. Esta variante descripta en Human Gene Mutation Database (HGMD), número de acceso ID CD961857, como asociada a un incremento en la susceptibilidad del riesgo de Cáncer de Mama y Ovario Hereditario. En el grupo de pacientes evaluados en el consejo genético y a los cuales se les solicitó test genético por sus antecedentes por cáncer de colon se detectó IMS-alta en 4 de los 31 casos $(12,9 \%)$, uno menor de 50 años y tres mayores de 50 años. Además, 11/31 (35,5\%), fueron inestables para D17S250, 3/31 (9,7\%) fueron inestables para D5S346, 2/31,(6,4 \%) fueron inestables para D2S123 y BAT 25, BAT 26 presentaron inestabilidad en $1 / 31(3,2 \%)$ (Tabla 2). No se detectaron rearreglos genómicos (pérdida ni ganancia de material genético) en los genes MLH1, MSH2, MSH6, PMS2 y regiones 3'de EPCAM por MLPA en los pacientes que presentaron IMS-alta (Tabla 3). El análisis de la mutación V600E del gen BRAF fue positivo en los cuatro pacientes con IMS-alta (Tabla 4). Los cuatro pacientes con cáncer de colon con resultados de los test genéticos positivos para CCR tenían antecedentes familiares de cáncer de colon. Los resultados de los estudios genéticos se informaron en forma personal a los efectos de responder a las preguntas y dudas que pudieran surgir y valorar el posible impacto psicológico. Se les explicó los resultados y se aseguró la comprensión de los mismos. También se establecieron planes para compartir los resultados con familiares, se le aclararon dudas se preservó la confidencialidad de los resultados y en algunos casos se ofreció apoyo psicológico. El control de las personas de alto riesgo se realizó en la unidad de consejo genético a los efectos del seguimiento de la misma y la familia a largo plazo, con actualización de la historia familiar y valoración de las modificaciones, revisación del control médico 
periódico y evaluación de las intervenciones solicitadas. El seguimiento de las personas con riesgo moderado y bajo se realizó en el Servicio de Oncología.

\section{Discusión}

Lo más importante a discutir es la utilidad de estos test en beneficio de la gente que concurre al consejo genético y la posibilidad que existe, en la realización del mismo, a los efectos de tomar recaudos a futuro con sus familias.

La prevalencia de las mutaciones $B R C A 1$ y $B R C A 2$ varía de acuerdo con el país y el grupo étnico ${ }^{5-9}$. En este estudio, en donde se secuenciaron los genes BRCA 1 y 2 completos en 34 mujeres obtuvimos un solo caso de BRCA 2 positivo. Entre los diferentes grupos étnicos la frecuencia más alta se encuentra en los individuos con ancestros judíosasquenazí ( 1 en 50) ${ }^{9}$. Otros grupos con alta prevalencia de mutaciones incluyen países como Islandia, Canadá (especialmente francocanadienses), Polonia y Holanda ${ }^{6}$. Estas altas tasas de prevalencia se deben a la presencia de mutaciones fundadoras, que son una o más mutaciones específicas en esa población que han sido heredadas de un ancestro común y que se han ido amplificando a través de las generaciones, contribuyendo a ello el aislamiento geográfico de la población. Por ejemplo, en la población judía asquenazí, existen dos mutaciones fundadoras en BRCA1 (185delAG y 5382insC) y una en BRCA2 (6174delT) que representan más de $90 \%$ de las mutaciones observadas en esta población ${ }^{9}$. Pero en países o regiones donde los orígenes de la población son mixtos debido a la variedad y a la mezcla de razas, siguen siendo necesarias más pruebas genéticas de secuenciación completa en ambos genes para poder determinar con precisión mutaciones fundadoras ${ }^{5-8}$. En este estudio la paciente que presento la mutación del BRCA 2 posee el fenotipo triple negativo y esto concuerda con los datos de la bibliografía, en donde los tumores de mama triple negativos, son tumores agresivos $\mathrm{y}$ en general los que presentan mutaciones en los genes BRCA 1 y $2^{10-12}$. Con respecto al estudio del cáncer de colon nosotros detectamos cuatro pacientes con IMS-alta y mutación del V600E del gen BRAF. Cuando se realizó el análisis de MLPA en los genes MSH6, MLH1, MSH2 y PMS2 en estos pacientes, a los efectos de establecer la diferencia entre CCR y síndrome de Lynch, los resultados fueron negativos y por lo tanto estos pacientes fueron diagnosticados como CCR esporádico. Mutaciones germinales en los genes MMR predisponen al cáncer colorrectal hereditario no polipósico (HNPCC) o síndrome de Lynch, que está presente en cerca del 3\% de todos los casos de CCR. Este síndrome es autosómico dominante, con alta penetrancia $(85 \%)$ y se caracteriza por un proceso acelerado de carcinogénesis ${ }^{13}$, por lo que se manifiesta en individuos menores de 50 años. La mayoría de las mutaciones ocurren en los genes MLH1 y MSH2 y se informan más de 500 mutaciones patogénicas: $50 \%$ en MLH1, $40 \%$ en MSH2 y $10 \%$ distribuidas entre los otros genes $\mathrm{MMR}^{14,15}$. Las mutaciones V600E en el gen BRAF ocurren aproximadamente en el $91 \%$ de los CCR esporádicos con IMS alta, pero no se observan en los pacientes con el síndrome de Lynch. Por lo anterior, es importante la determinación de mutaciones del gen $B R A F$ en pacientes con CCR, porque permite distinguir subgrupos de tumores con IMS-alta de tipo esporádico de los del síndrome de Lynch $^{16,17}$. Además, estudios recientes informan que el gen $B R A F$ podría considerarse como un biomarcador predictivo $\mathrm{y}$ pronóstico, puesto que los pacientes con $B R A F$ mutado no tienen una buena respuesta a los tratamientos basados en EGFR-ITK y, además, presentan mal pronóstico ${ }^{18,19}$.

\section{Conclusiones}

Como lo demuestra este trabajo, en el consejo genético, el estudio de vías moleculares en pacientes con cáncer hereditario es un instrumento de ayuda para la valoración del riesgo, recomendando pautas de actuación en los ámbitos de diagnóstico, prevención y tratamiento sobre la base del nivel de evidencia científica existente.

En este estudio los marcadores moleculares BRCA 1 y 2, IMS, MSH6, MLH1, MSH2, PMS2 y $B R A F$, nos permitieron definir las conductas a seguir. Por lo tanto consideramos importante la implementación de las pruebas moleculares a los efectos de establecer un diagnóstico genético definitivo.

\section{Agradecimientos}

A la Licenciada en Genética Celeste Goy por la lectura crítica del trabajo. Proyecto que fue aceptado y evaluado en las convocatorias 2013 2016 de la Secretaria de Investigación de la UCC.

\section{Bibliografía}

1. Evans DG, Lalloo F. Risk assessment and management of high risk familial breast cancer. J Med Genet. 2002;39(12):865-71.

2. Richards, S. Aziz, N. Bale, S. Bick, D. etal. Standards and guidelines for the interpretation of sequence variants: a joint consensus 
recommendation of the American College of Medical Genetics and Genomics and the Association for Molecular Pathology. Genet. Med.2015.17,405-24

3. Human Genome Variation Society Guidelines \& Recommendations. Disponible en http://www.hgvs.org/content/guidelines.

4. Boland CR, Thibodeau SN, Hamilton SR, Sidransky D, et al. National Center Institute Workshop on Microsatellite Instability for cancer detection and familial predisposition: development of international criteria for the determination of microsatellite instability in colorectal cancer. Cancer Res. 1998;58:524857.

5. Hall MJ, Reid JE, Burbidge LA, Pruss D, et al. Women of Different Ethnicities Undergoing Testing for Hereditary BreastOvarian Cancer. Cancer 2009;115(10):22222233.

6. Cazap E, Buzaid AC, Garbino C, de la Garza $\mathrm{J}$, et al. Latin American and Caribbean Society of MedicalOncology. Breast cancer in Latin America: results of the Latin Americanand Caribbean Society of Medical Oncology/Breast Cancer ResearchFoundation expert survey. Cancer $2008 ; 113(8$ Suppl):2359-2365.

7. John EM, Miron A, Gong G, Phipps AI, et al. Prevalenceof pathogenic BRCAl mutation carriers in 5 US racial groups. JAMA 2007;298:2869-2876.

8. Malone K, Daling J, Doody D, Hsu L, et al.Prevalence and Predictors of BRCAl and BRCA2 Mutations in aPopulation-Based Study of Breast Cancer in White and Black AmericanWomen Ages 35 to 64 Years. Cancer Res 2006; 66(16): 8297-8308.

9. Warner E, Foulkes W, Goodwin P, Meschino $\mathrm{W}$, et al. Prevalence and penetrance of BRCA1 and BRCA2 gene mutations inmunoselected Ashkenazi Jewish women with breast cancer.J NatlCancer Inst1999;91:1241-1247.

10. Evans DG, Howell A, Ward D, Gragan,Het al. Prevalence of BRCA1 and BRCA2 mutations

in triple negative breast cancer. J Med Genet. 2011 Aug;48(8):520-2

11. Young SR, Pilarski RT, Donenberg T,Gorusku, $\mathrm{M}$ et al. The prevalence of $B R C A 1$ mutations among young women with triplenegative breast cancer.BMC Cancer. 2009 Mar 19;9:86.

12. Rummel S, Varner E, Shriver CD, Harrinson,Jet al. Evaluation of BRCA1 mutations in an unselected patient population with triple-negative breast cancer. Breast Cancer Res Treat. 2013 Jan;137(1):119-25.

13. Godai TI, Suda T, Sugano N, Tsuchida K, et al.Identification of colorectal cancer patients with tumors carrying the TP53 mutation on the codon 72 proline allele that benefited most from 5-fluorouracil (5-FU) based postoperative chemotherapy. BMC Cancer. 2009;9:420

14. Calvacanti-Carneiro da Silva F, DominguezValentin M, de Oliveira-Ferreira F, CarraroDM, et al. Mismatch repair genes in Lynch syndrome: a review. Sao Paulo Medical Journal. 2009;127(1):46-51.

15. Hsieh P, Yamane K. DNA mismatch repair: molecular mechanism, cancer, and ageing.Mech Ageing Dev. 2008 JulAug;129(7-8):391-407.

16. Sharma SG, Gulley ML. B-RAF mutation testing in colorectal cancer. Arch Pathol Lab Med. 2010 Aug;134(8):1225-8.

17. Rozek LS, Herron CM, Greenson JK, Moreno $\mathrm{V}$, et al. Smoking, gender, and ethnicity predict somatic $B-R A F$ mutations in colorectal cancer. Cancer Epidemiol Biomarkers Prev. 2010 Mar;19(3):838-43.

18. Prenen H, Tejpar S, Van Cutsem E. Impact of molecular markers on treatment selection in advanced colorectal cancer. Eur J Cancer. 2009 Sep;45Suppl 1:70-8.

19. Ogino S, Nosho K, Kirkner GJ, Kawasaki T, et alCpG island methylator phenotype, microsatellite instability, $B-R A F$ mutation and clinical outcome in colon cancer. Gut. 2009 Jan;58(1):90-6.

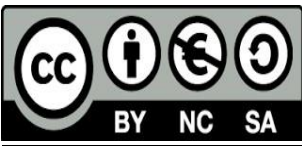




\section{Anexo tablas}

Tabla 1. Historia familiar de cáncer de mama/ovario y $B R C A 1$ - BRCA 2

\begin{tabular}{|c|c|c|c|c|c|}
\hline \multicolumn{2}{|c|}{ Paciente/edad } & \multirow{2}{*}{$\begin{array}{l}\text { BRCA } 1 \\
\text { Negativo }\end{array}$} & \multirow{2}{*}{$\begin{array}{l}\text { BRCA } 2 \\
\text { Negativo }\end{array}$} & \multirow{2}{*}{$\begin{array}{l}\text { Tumor- } \\
\text { Fenotipo } \\
\text { Triple negativo }\end{array}$} & \multirow{2}{*}{$\begin{array}{l}\text { Historia } \\
\text { Familiar } \\
\mathrm{Si}\end{array}$} \\
\hline 1 & 32 & & & & \\
\hline 2 & 37 & Negativo & Negativo & Triple negativo & $\mathrm{Si}$ \\
\hline 3 & 40 & Negativo & Negativo & Triple negativo & Si \\
\hline 4 & 36 & Negativo & Negativo & Luminal A & $\mathrm{Si}$ \\
\hline 5 & 46 & Negativo & Negativo & Triple negativo & $\mathrm{Si}$ \\
\hline 6 & 54 & Negativo & Negativo & Triple negativo & $\mathrm{Si}$ \\
\hline 7 & 50 & Negativo & Positivo & Triple negativo & $\mathrm{Si}$ \\
\hline 8 & 61 & Negativo & Negativo & $\begin{array}{ll}\begin{array}{l}\text { Cancer } \\
\text { ovario }\end{array} & \text { de } \\
\end{array}$ & $\mathrm{Si}$ \\
\hline 9 & 36 & Negativo & Negativo & Triple negativo & Si \\
\hline 10 & 58 & Negativo & Negativo & Triple negativo & $\mathrm{Si}$ \\
\hline 11 & 48 & Negativo & Negativo & Triple negativo & $\mathrm{Si}$ \\
\hline 12 & 33 & Negativo & Negativo & Triple negativo & Si \\
\hline 13 & 33 & Negativo & Negativo & Luminal $\mathrm{A}$ & $\mathrm{Si}$ \\
\hline 14 & 47 & Negativo & Negativo & Triple negativo & $\mathrm{Si}$ \\
\hline 15 & 35 & Negativo & Negativo & Triple negativo & $\mathrm{Si}$ \\
\hline 16 & 41 & Negativo & Negativo & Luminal $\mathrm{A}$ & $\mathrm{Si}$ \\
\hline 17 & 42 & Negativo & Negativo & Triple negativo & $\mathrm{Si}$ \\
\hline 18 & 40 & Negativo & Negativo & $\begin{array}{l}\text { Cáncer } \\
\text { ovario }\end{array}$ & Si \\
\hline 19 & 28 & Negativo & Negativo & Triple negativo & $\mathrm{Si}$ \\
\hline 20 & 32 & Negativo & Negativo & Luminal $\mathrm{A}$ & $\mathrm{Si}$ \\
\hline 21 & 54 & Negativo & Negativo & Triple negativo & $\mathrm{Si}$ \\
\hline 22 & 42 & Negativo & Negativo & Luminal A & $\mathrm{Si}$ \\
\hline 23 & 43 & Negativo & Negativo & $\begin{array}{l}\text { Cáncer } \\
\text { ovario }\end{array}$ & $\mathrm{Si}$ \\
\hline 24 & 74 & Negativo & Negativo & Triple negativo & $\mathrm{Si}$ \\
\hline 25 & 36 & Negativo & Negativo & $\begin{array}{l}\text { Cáncer } \\
\text { ovario }\end{array}$ & $\mathrm{Si}$ \\
\hline 26 & 38 & Negativo & Negativo & Triple negativo & $\mathrm{Si}$ \\
\hline 27 & 43 & Negativo & Negativo & Triple negativo & $\mathrm{Si}$ \\
\hline 28 & 33 & Negativo & Negativo & $\begin{array}{l}\text { Cáncer } \\
\text { ovario }\end{array}$ & Si \\
\hline 29 & 52 & Negativo & Negativo & Triple negativo & $\mathrm{Si}$ \\
\hline 30 & 43 & Negativo & Negativo & Triple negativo & $\mathrm{Si}$ \\
\hline 31 & 51 & Negativo & Negativo & Luminal $\mathrm{A}$ & $\mathrm{Si}$ \\
\hline 32 & 41 & Negativo & Negativo & Triple negativo & $\mathrm{Si}$ \\
\hline 33 & 41 & Negativo & Negativo & $\begin{array}{l}\text { Cáncer } \\
\text { ovario }\end{array}$ & Si \\
\hline 34 & 49 & Negativo & Negativo & Triple negativo & Si \\
\hline
\end{tabular}


Tabla 2. Inestabilidad de microsatélites

\begin{tabular}{|c|c|c|c|c|c|c|c|c|}
\hline \multicolumn{9}{|c|}{ Marcadores microsatelitales del Panel de Bethseda } \\
\hline Pacientes & Edad/S & & $\begin{array}{l}\text { BAT } \\
25\end{array}$ & $\begin{array}{l}\text { BAT } \\
26\end{array}$ & D5S346 & D17S250 & D2S123 & Resultados \\
\hline 1 & $33-F$ & $E$ & & $\bar{E}$ & $\bar{E}$ & $\bar{E}$ & $\bar{E}$ & EMS \\
\hline 2 & $66-M$ & $E$ & & $E$ & $E$ & T & $E$ & IMS-L \\
\hline 3 & $62-M$ & $E$ & & $E$ & E & $E$ & $E$ & EMS \\
\hline 4 & $56-M$ & $E$ & & $E$ & $E$ & $E$ & $E$ & EMS \\
\hline 5 & $26-F$ & $E$ & & $E$ & $E$ & $\mathrm{LOH}$ & $\mathrm{LOH}$ & CIN \\
\hline 6 & $68-M$ & $E$ & & $E$ & $E$ & I & $E$ & IMS-L \\
\hline 7 & $67-M$ & $E$ & & $E$ & E & I & $E$ & IMS-L \\
\hline 8 & $61-F$ & $E$ & & $E$ & $E$ & $E$ & $E$ & EMS \\
\hline 9 & $61-M$ & $E$ & & $E$ & $E$ & $E$ & $E$ & EMS \\
\hline 10 & $48-M$ & $E$ & & $E$ & $E$ & I & $E$ & IMS-L \\
\hline 11 & 71-M & $E$ & & $E$ & $E$ & I & $E$ & IMS-L \\
\hline 12 & $55-F$ & $E$ & & $E$ & I & $E$ & I & IMS-H \\
\hline 13 & $70-F$ & $E$ & & $E$ & $E$ & $E$ & $E$ & EMS \\
\hline 14 & $66-F$ & $E$ & & $E$ & $E$ & $E$ & $E$ & EMS \\
\hline 15 & $64-M$ & $E$ & & $E$ & $E$ & $E$ & $E$ & EMS \\
\hline 16 & $71-M$ & $E$ & & $E$ & $E$ & I & $E$ & IMS-L \\
\hline 17 & $57-\mathrm{M}$ & $E$ & & $E$ & I & $E$ & I & IMS-H \\
\hline 18 & $58-F$ & $E$ & & $E$ & $E$ & $E$ & $E$ & EMS \\
\hline 19 & $68-F$ & $E$ & & $E$ & $E$ & $E$ & $E$ & EMS \\
\hline 20 & $34-\mathrm{M}$ & I & & I & $E$ & $E$ & $E$ & IMS-H \\
\hline 21 & $77-\mathrm{F}$ & $E$ & & $E$ & $E$ & $E$ & $E$ & EMS \\
\hline 22 & $58-F$ & $E$ & & $E$ & $E$ & $E$ & $E$ & EMS \\
\hline 23 & $62-M$ & $E$ & & $E$ & I & I & $E$ & IMS-H \\
\hline 24 & $65-M$ & $E$ & & $E$ & $E$ & $E$ & $E$ & EMS \\
\hline 25 & $56-\mathrm{M}$ & $E$ & & $E$ & E & $E$ & $E$ & EMS \\
\hline 26 & $70-M$ & $E$ & & $E$ & $E$ & $E$ & $E$ & EMS \\
\hline 27 & $59-M$ & $E$ & & $E$ & E & I & $E$ & IMS -L \\
\hline 28 & $71-M$ & $E$ & & $E$ & $E$ & $E$ & $E$ & EMS \\
\hline 29 & $70-\mathrm{M}$ & $E$ & & $E$ & E & I & $E$ & IMS -L \\
\hline 30 & $54-F$ & $E$ & & $E$ & $E$ & I & $E$ & IMS-L \\
\hline 31 & $68-F$ & $E$ & & $E$ & $E$ & I & $E$ & IMS-L \\
\hline
\end{tabular}

CIN: inestabilidad cromosómica, LOH: pérdida de heterocigosidad, EMS: estable, IMS-L: leve, IMS-H: alta.

Tabla 3. Rearreglosgenómicos por MLPA

\begin{tabular}{lllllll}
\hline Pacientes & Edad/sexo & MLH1 & MSH2 & MSH6 & PMS2 & 3' EPCAM \\
\hline $\mathbf{1 2}$ & $55-\mathrm{F}$ & Negativo & Negativo & Negativo & Negativo & Negativo \\
$\mathbf{1 7}$ & $57-\mathrm{M}$ & Negativo & Negativo & Negativo & Negativo & Negativo \\
$\mathbf{2 0}$ & $\mathbf{3 4 - \mathrm { M }}$ & Negativo & Negativo & Negativo & Negativo & Negativo \\
$\mathbf{2 3}$ & $62-\mathrm{M}$ & Negativo & Negativo & Negativo & Negativo & Negativo \\
\hline
\end{tabular}


Lerda D, Pellicioni P, Biaggi Bioq M, Labrador J, Illescas E, Bella S, Llugdar J, Cortes M. Consejo genético y detección de vías moleculares en pacientes con cáncer hereditario.

Tabla 4. Mutación V600E BRAF

\begin{tabular}{llll}
\hline Pacientes & Edad/sexo & V600E BRAF \\
\hline $\mathbf{1 2}$ & $55-\mathrm{F}$ & Mutado \\
$\mathbf{1 7}$ & $57-\mathrm{M}$ & Mutado & \\
$\mathbf{2 0}$ & $34-\mathrm{M}$ & Mutado & \\
$\mathbf{2 3}$ & $62-\mathrm{M}$ & Mutado \\
\hline
\end{tabular}

\section{(c) (1)(0) \\ BY NC SA}

